\title{
Corela
}

Cognition, représentation, langage

13-1 | 2015

Vol. $13, \mathrm{n}^{\circ} 1$

\section{Variation sur une coupure : "short of » et « shy of », entre division et discernement}

\author{
Philippe Muller
}

\section{OpenEdition}

\section{Journals}

Édition électronique

URL : http://journals.openedition.org/corela/3983

DOI : $10.4000 /$ corela.3983

ISSN : $1638-573 \mathrm{X}$

\section{Éditeur}

Cercle linguistique du Centre et de l'Ouest - CerLICO

\section{Référence électronique}

Philippe Muller, " Variation sur une coupure : "short of » et "shy of », entre division et discernement », Corela [En ligne], 13-1 | 2015, mis en ligne le 30 juin 2015, consulté le 01 mai 2019. URL : http:// journals.openedition.org/corela/3983 ; DOI : 10.4000/corela.3983

Ce document a été généré automatiquement le 1 mai 2019.

\section{(c) (i) (2)(2)}

Corela - cognition, représentation, langage est mis à disposition selon les termes de la licence Creative Commons Attribution - Pas d'Utilisation Commerciale - Partage dans les Mêmes Conditions 4.0 International. 


\title{
Variation sur une coupure : "short of " et "shy of ", entre division et discernement
}

\author{
Philippe Muller
}

Je tiens à remercier Mme Hélène Chuquet et deux relecteurs anonymes pour leurs remarques sur ce texte. Je suis bien sûr seul responsable de ses imperfections.

\section{0 . De SHY à SHORT OF : contexte, objectifs et cadre de cet article}

1 Cet article se veut le prolongement de celui consacré au lexème SHY dans lequel j'ai proposé pour ce terme une représentation topologique dans une optique heuristique ( $\mathrm{P}$. Muller (2015a)). Il s'agissait à travers celle-ci de contribuer à cerner son identité linguistique dans l'optique plus lointaine de dégager ce que la TOPE nomme sa «forme schématique ». Sans revenir longuement sur ce concept déjà présenté dans mon travail précédent, j'y fais appel essentiellement car il s'appuie sur une conception de l'invariance dégagée par abstraction des différents contextes et qui met en valeur à la fois une certaine stabilité et une certaine déformabilité de la description métalinguistique proposée. Il permet de ne pas envisager la primauté sémantique de tel ou tel emploi en discours, chacune des «valeurs» particulières prise par la forme linguistique dans l'énoncé n'étant qu'une réalisation empirique possible de cette forme schématique. Par ailleurs, il est possible que seuls certains aspects en soient activés le cas échéant (voir D. Paillard et J.-J. Franckel (2007)).

2 Je commencerai par rappeler ici l'hypothèse générale envisagée pour SHY avant de justifier la représentation intuitive de SHORT OF que j'ai été amené à proposer à la toute fin de mon article précédent. Je testerai alors sa pertinence en la confrontant à un corpus constitué de quelques-uns des 387 énoncés de la section «newspaper» du $\mathrm{BNC}^{1}$ dans 
lesquels cette construction apparait. Je proposerai au passage une hypothèse pour SHORT lui-même, à savoir qu'il permet de construire ce que l'on peut appeler la "portion congrue » d'un domaine notionnel.

Tout au long de cet article, j'opposerai au niveau notionnel SHY OF et SHORT OF en faisant du premier un marqueur de «discernement» et du second un marqueur de «division », m'inspirant ainsi de D. Paillard (1998), analyse que j'ai esquissée dans P. Muller (2015b). Je montrerai aussi que le concept d'« intentionnalité » que l'on doit à J.-J. Franckel et D. Lebaud (1990), permet de rendre compte de SHY OF et d'établir pour ce marqueur une certaine congruence entre les niveaux d'analyse ${ }^{2}$ notionnel et énonciatif. Cette congruence n'existe pas a priori avec SHORT OF, le marqueur de division ne favorisant pas en soi la construction de l'intentionnalité. Néanmoins, certains contextes particuliers permettent ce type d'interprétation énonciative et relativisent l'opposition SHY OF / SHORT OF, ce qui pousse à réanalyser notionnellement le marqueur de division en marqueur de discernement. L'énonciateur invite alors le co-énonciateur à suivre un cheminement interprétatif qui n'en reste pas moins différent avec les deux constructions.

\section{Rappel et approfondissement de l'analyse proposée pour SHY}

4 Cette première partie qui reprend l'analyse proposée dans mes deux articles précédents sera nécessairement moins démonstrative en comparaison. Elle vise plutôt à compléter et à approfondir les implications théoriques des hypothèses déjà posées pour SHY et, accessoirement, pour SHORT OF, afin de faire émerger des éléments d'opposition plus précis qui me permettront de développer l'analyse de SHORT OF que je n'avais pas pu exposer jusqu'à présent.

\subsection{SHY COMME MARQUE D'UN HIATUS ENTRE DEUX TERMES X ET Y}

5 L'hypothèse que j'ai proposée pour SHY était que ce lexème se laisse décrire comme un marqueur signalant une opération de localisation ${ }^{3}$ entre deux termes $\mathrm{X}$ et $\mathrm{Y}$ telle que $\mathrm{X}$ acquiert une détermination qualitative en vertu du fait qu'il est donné comme extérieur à $\mathrm{Y}$ tout en restant dans son voisinage. La qualité ainsi attribuée à X que j'ai appelée par commodité "shyness» émerge en fait conjointement à la construction d'un " hiatus " entre $\mathrm{X}$ et $\mathrm{Y}$, et ceci que l'enjeu global de l'énoncé soit plutôt qualitatif, par exemple coder le sentiment de timidité (SHY adjectival (1)), ou plutôt quantitatif , coder un mouvement de recul spatio-temporel qui peut être plus ou moins volontaire (SHY verbal (2)) :

(1) $[\mathrm{He}]_{\mathrm{X}}$ seems in no hurry to start a family of his own and is shy about [discussing beautiful Kristy $]_{Y}$, who he has been linked with romantically. (CH1)

(2) Second, [they $]_{\mathrm{X}}$ put themselves on the defensive by shying away from [subjects

like food scares $]_{Y}$ and by protecting the rogues within their industry. (HJ3)

6 Par «hiatus », il faut entendre une interruption, ou une coupure, dans les repérages spatio-temporels ${ }^{4}$ permettant de construire un intervalle à bornes distinctes qui matérialise une béance plus ou moins quantifiable entre deux termes (voir la représentation topologique proposée en 1.5). Si cette interruption est évidente dans le cas du SHY verbal qui impose une distanciation physique entre $\mathrm{X}$ et $\mathrm{Y}$, on peut proposer, afin de transcender les variations contextuelles, qu'elle est aussi pertinente dans l'usage adjectival, où il s'agit de renvoyer à un sentiment de timidité (voir 1.2). 
7 Même lorsque le terme $\mathrm{Y}$ n'est pas instancié en surface, l'hiatus avec X s'établit à partir d'un second terme implicite, la sociabilité, ou ce que l'on peut appeler en anglais « social interaction » à la suite des psychologues P. Manning et G. Ray (1993). SHY marque alors un écart par rapport à une sociabilité généralement valorisée par la norme interpersonnelle :

(3) Off the judo mat, [Howey $]_{x}$ is reserved and even shy. (AJR)

Lorsque $Y$ est instancié, l'hypothèse de l'hiatus semble rester pertinente quelle que soit la préposition avec laquelle SHY se construit (OF, FROM, ABOUT, AWAY (FROM), etc...), même si l'on peut supposer que chacune d'elles apporte une modulation particulière sur la réalisation empirique de la forme schématique. Je considérerai en tout cas ici que la représentation qu'on peut donner de SHY ne diffère pas fondamentalement de celle que l'on peut donner de SHY OF, selon cette hypothèse qui fait qu'en anglais moderne on est toujours fondamentalement «SHY OF SOMETHING». Il faudrait affiner l'analyse et distinguer dans un autre travail ce qui appartient à SHY et ce qui appartient à la préposition.

Afin de réfléchir à l'identité du lexème, il apparaît en tout cas avantageux de généraliser l'idée d'un hiatus. On peut en fait penser que celui-ci émerge parce que SHY permet de construire le terme $\mathrm{X}$ comme différencié qualitativement du terme $\mathrm{Y}$.

\subsection{SHY COMME « OPÉRATEUR DE DISCERNEMENT » QUALITATIF}

10 Au niveau notionnel, SHY fait office de marqueur de "discernement ", pour emprunter à J.-J. Franckel et D. Paillard (2007: 8), ce qui signifie « que $Y$ attribue des propriétés non définitoires au terme $\mathrm{X}$ mis en relation à $\mathrm{Y}[. .$.$] et constitue un mode d'appréhension de$ $\mathrm{X} »$.

11 C'est ce qui est exploité de manière très sensible à travers les composés adjectivaux en SHY comme « $X$ is girl-shy / work-shy / love-shy, etc... » qui permettent d'appréhender qualitativement $\mathrm{X}$ en relation avec le terme $\mathrm{Y}$ (girl, work ou love), dans la mesure où celuici participe à en cerner l'identité. Le corpus étudié contient un exemple de ce type :

(4) [Publicity $]_{\underline{Y}}$ shy $[\text { Anne }]_{X}$, who tried to keep details of her wedding secret, set out with black gloves to hide the sparkler. (CBF)

On obtient un mode d'appréhension de $\mathrm{X}$ qui pourrait se laisser gloser par « dis-moi qui tu fuis et je te dirai qui tu es ». Dans cette hypothèse, l'enjeu principal avec SHY, est le discernement de l'identité de $\mathrm{X}$ sous le mode de la dissociation par rapport à un terme $\mathrm{Y}$ plus ou moins explicite, et ceci que le contexte général d'emploi soit à dominante " émotionnelle » ou "spatio-temporelle ». Le terme de « dissociation » se justifie dans la mesure où SHY marque que l'énonciateur envisage la déconstruction d'une association sous-jacente entre les deux termes, c'est à dire l'interruption d'une certaine continuité qualitative entre les deux termes, comme le montrera l'analyse des énoncés à interprétation spatio-temporelle (voir 1.4). Notons pour l'instant que le sentiment de timidité lui-même est intuitivement conceptualisable à travers la disjonction de deux termes, ce qui aurait pour conséquence un « recul » physique plus ou moins réel de X par rapport à $Y$ (=les relations sociales). Cette séparation spatio-temporelle établie entre les deux termes est significative sur le plan qualitatif et il s'agit bien d'un mode d'appréhension de X. La glose donnée par l'OED le montre bien, SHY signifiant « shrinking from self-assertion; sensitively timid; retiring or reserved from diffidence; bashful ». Le mot « retiring » apparaît d'ailleurs clairement en (5) : 
(5) A Certain Sacrifice, made in 1979, features the shy, retiring lady we later came to

know as Madonna in a drama set on New York's meanest streets. (K97)

\subsection{MOdÉLISATION DE L'HIATUS MARQUÉ PAR SHY À TRAVERS LE COUPLE QNT / QLT}

16 On peut modéliser l'hiatus marqué par SHY à travers la dichotomie devenue classique dans la TOPE entre des repérages relevant des paramètres QNT et QLT. De façon générale, le premier est ce qui permet la fragmentation de la notion en occurrences et le fait de situer celles-ci « dans l'espace-temps énonciatif qu'un sujet énonciateur situe par rapport à un co-énonciateur » (A. Culioli, 1999b : 5). Le second permet d'organiser la cohésion de la notion ou des occurrences de notion autour d'un faisceau de propriétés physicoculturelles et « entre en jeu chaque fois que l'on effectue une opération d'identification / différenciation » (Ibid : 6). Récemment, L. Dufaye (2009 : 94-100) a proposé de distinguer, au sein de ce couple, des repérages extra- et intra-notionnels. On peut ainsi reprendre cette distinction et envisager le repérage entre $\mathrm{X}$ et $\mathrm{Y}$ à travers quatre paramètres :

-QNT ${ }_{1}$ (extra-notionnel) : l'existence quantitative des occurrences est conceptualisée de façon externe. La prédication SHY qui relie les deux termes $\mathrm{X}$ et $\mathrm{Y}$ renvoie à une notion complexe dont des occurrences sont inscriptibles dans l'espace-temps. Il peut s'agir d'un procès (7), d'une relation adjectivale (8), voire prépositionnelle (voir 1.4 pour ces exemples, du type «In 1837, the Edwards Dam was built across the Kennebec River, just shy of the limit of tidal influence» (Internet)) :

(7) Time and time again [horses $]_{X}$ shy away from [the whip $]_{Y}$ and lose races. (K5A)

(8) The scouts do exciting and hazardous things, but [they] ${ }_{X}^{\prime}$ 're sometimes shy about [admitting that they're in the scouts $]_{\mathrm{Y}}$ (K1H) 
QNT $_{1}$ autorise à concevoir l'ensemble de la prédication construite par SHY comme renvoyant à une situation de référence unique dont on peut envisager globalement le passage à l'existence bien qu'elle mette en jeu des entités séparées.

- $\mathrm{QNT}_{2}$ (intra-notionnel) : permet de prendre en compte des discontinuités et des intervalles distincts à l'intérieur du domaine notionnel. Les deux termes $\mathrm{X}$ et $\mathrm{Y}$ reliés par SHY renvoient à deux existants distincts, non confondables dans l'espace-temps. Poser qu'il y a, avec SHY, rupture dans les repérages $\mathrm{QNT}_{2}$, c'est-à-dire prise en compte d'une « coupure sans continuité sous-jacente» (L. Dufaye, ibid: 89), permet de modéliser la construction d'une distance entre $\mathrm{X}$ et $\mathrm{Y}$ :

(9) The plan started to work. Within a few days $[\text { he }]_{X}$ became shy and nervous of

[humans] $]_{\mathrm{Y}}$ even at feeding times. (K1V)

$\mathrm{X}$ et $\mathrm{Y}$ ne sont pas totalement indépendants l'un de l'autre, mais sont à la fois rassemblés et maintenus « éloignés " au niveau prédicatif par SHY qui fonctionne comme un relateur 7. SHY joue un rôle tout aussi paradoxal lorsque l'on se place à un niveau notionnel d'analyse. Il réunit ainsi globalement dans l'espace-temps ( $\left.\mathrm{QNT}_{1}\right)$ deux termes $\mathrm{X}$ et $\mathrm{Y}$ qu'il sépare par ailleurs via la construction d'un hiatus. Ce hiatus est obtenu par un repérage qui repose sur une opération de rupture c'est-à-dire la prise en compte d'une altérité forte du point de vue de $\mathrm{QNT}_{2}$.

21 Cependant, et sur un plan qualitatif, il ne s'agit pas simplement de rejeter X dans l'altérité pensée à partir de $\mathrm{Y}$ car ce rejet s'accompagne de l'établissement d'une tension entre $\mathrm{X}$ et Y, ce que l'on peut modéliser au niveau notionnel à partir des repérages QLT.

- QLT $_{1}$ (extra-notionnel): l'existence qualitative des occurrences est envisagée globalement. La question de la cohésion notionnelle de l'ensemble X-relateur-Y est appréhendable de manière externe à la prédication. SHY maintient une certaine homogénéité d'ensemble en ce qu'il construit une scène cognitive globale opposable à d'autres scènes cognitives possibles qui organiseraient le repérage entre $X$ et $Y$ sur un mode différent :

(10) Characters are apparently motiveless, [compulsive liars]X, who shy from [eye contact]Y. (A4A)

23 Le verbe SHY est ici à la fois proche et différent du verbe AVOID, par exemple. Il s'agit d'envisager ce qui fait la spécificité, ou l'identité, du domaine notionnel complexe car, comme l'écrit G. Mélis (2004 :79), QLT 1 permet « un travail identificatoire [...] dont la fonction est de reconnaître une occurrence en la distinguant des autres sur le plan de son identité propre. »

24 - $\mathrm{QLT}_{2}$ (intra-notionnel) : la problématique de la cohésion est envisageable de manière interne, ce qui permet d'aborder les questions de l'identité, de la variation et du changement à l'intérieur du domaine notionnel complexe. On effectue alors « un travail de spécification [...] qui consiste à donner une meilleure définition d'un état de fait déjà identifié qui peut donner lieu à un contraste avec d'autres valeurs, ou à introduire une qualification commentative » (Mélis, Ibid : 79).

On peut penser pour SHY que $X$ et $Y$ sont envisagés par une opération de différenciation au niveau de $\mathrm{QLT}_{2}$, c'est-à-dire une «double opération de repérage par laquelle on renvoie à la fois à de l'identification et à de l'altérité » (L. Dufaye, 2009 : 85). Avec SHY, on a l'établissement d'une tension dynamique entre les deux termes, et ceci même dans un emploi de type adjectival : 
(11) [ULSTER spacers $]_{\mathrm{X}}$ have been shy about [signing up for inter-galactic travel] $]_{\mathrm{Y}}$. (K2D)

Ce qui fait exister $\mathrm{X}$ en tant que $\mathrm{X}$, c'est le fait d'être appréhendé comme existant dans le prolongement de $\mathrm{Y}$ auquel il est à la fois partiellement assimilé et duquel il est différencié. C'est ce qui fonde l'analyse de SHY en marqueur de discernement opérant sur une dissociation $\mathrm{X} / \mathrm{Y}$.

En résumé, l'hypothèse proposée est que SHY marque :

- une différenciation $\mathrm{QLT}_{2}$ entre $\mathrm{X}$ et $\mathrm{Y}$ qui entraînerait

- une rupture $\mathrm{QNT}_{2}$ établissant un hiatus plus ou moins quantifiable entre les deux termes.

L'identification sous-jacente QLT $_{2}$ impliquée par la première opération pousserait à une relativisation de l'hiatus quantitatif entre X et $\mathrm{Y}$. J'ai justifié cette hypothèse (P. Muller, (2015b)) à partir de l'étude des effets de sens qui sont attachés à la construction SHY OF, lorsqu'elle peut commuter avec SHORT OF - donc dans un emploi spatio-temporel. Je rappelle brièvement cette analyse.

\subsection{SHY OF spatio-temporel. La question d'une identification $\mathrm{QLT}_{2}$ sous jacente}

L'étude des énoncés du BNC conduit à remarquer que de nombreux contextes où SHY apparaît intègrent l'idée que l'hiatus postulé est en fait annulable et que cela est même le plus souvent souhaité par l'énonciateur, notamment lorsque SHY OF peut commuter avec SHORT OF, c'est-à-dire lorsque l'on est en présence de ce qui s'apparente plutôt à une préposition complexe, D. Thatcher (2014) suggérant leur interchangeabilité. On peut pourtant défendre l'idée que SHY OF a la particularité d'imposer une continuité qualitative "malgré tout », entre $\mathrm{X}$ et $\mathrm{Y}$ éloignés dans la situation de référence ${ }^{8}$, ce qui permet d'envisager implicitement l'annulation éventuelle de l'hiatus spatio-temporel. Une nuance de sens est en effet perceptible entre (12) et (13) où l'hiatus lui-même est quantifié par un terme $\mathrm{Z}$ :

(12) $[\text { Fred }]_{X}$, who died before Christmas [only a month $]_{Z}$ shy of [93 $]_{Y}$, had a rich fund

of stories, most of them against himself. (HJ3)

(13) $[\text { Fred }]_{\mathrm{X}}$, who died before Christmas [only a month $]_{\mathrm{Z}}$ short of $[93]_{\mathrm{Y}}$, had a rich

fund of stories, most of them against himself.

L'énoncé en SHY OF s'interprète en effet comme la manifestation de l'adversité, voire du destin et l'énonciateur regrette le maintien de l'hiatus quantifié par $\mathrm{Z}$, ce qui signifie qu'un autre possible était attendu dans la situation de référence : l'accession pré-envisagée de $\mathrm{X}$ au domaine de $\mathrm{Y}$. (12) marque qu'il était préconstruit que Fred (X) parvienne à atteindre l'âge de 93 ans (Y) mais qu'il a échoué en chemin. Cette interprétation est moins immédiatement disponible en (13). On a avec SHY l'interruption d'une identification qualitative sous-jacente entre $\mathrm{X}$ et $\mathrm{Y}$. Il semble d'ailleurs plus facile d'omettre l'adverbe ONLY en (13) qu'en (12), ce qui confirme l'analyse de SHY comme une marque d'appréciation subjective d'un état de $\mathrm{X}^{9}$.

En (14), remplacer SHY par SHORT gomme l'idée qu'il était a priori souhaitable, et surtout possible, que les actions ( $\mathrm{X}=$ the shares) atteignent le prix escompté ( $\mathrm{Y}=$ the float price). Les deux termes sont alors inconciliables et sont appréhendés comme essentiellement différents et immuablement distants l'un de l'autre, cette distance étant quantifiée par Z : 
(14) But the deepening recession in most continental countries is hitting sales across the Channel. [The shares] $]_{\mathrm{X}}$ added $1 \mathrm{p}$ to $256 \mathrm{p},[29 \mathrm{p}]_{\mathrm{Z}}$ shy of [the float price $]_{\mathrm{Y}}$. (CBD)

(15) [The shares $]_{\mathrm{X}}$ added $1 \mathrm{p}$ to $256 \mathrm{p},[29 \mathrm{p}]_{\mathrm{Z}}$ short of [the float price $]_{\mathrm{Y}}$.

Avec SHY on peut s'attendre à ce que l'hiatus soit quantitativement réduit à zéro car l'identification $\mathrm{QLT}_{2}$ entraîne sur le plan spatio-temporel une relativisation de la rupture du repérage $\mathrm{QNT}_{2}$ entre $\mathrm{X}$ et $\mathrm{Y}$. SHY OF peut alors s'analyser comme marquant le caractère non effectif d'une accession pré-envisagée de $\mathrm{X}$ à $\mathrm{Y}$, ce qui permet le discernement qualitatif de X par Y sur le mode de la dissociation. On peut penser que c'est cette dissociation au niveau de $\mathrm{QLT}_{2}$ qui entraîne la construction de l'hiatus au niveau de $\mathrm{QNT}_{2}$.

Dans cette analyse, la rupture QNT que SHY OF marque entre X et $\mathrm{Y}$ reste donc subordonnée à la détermination QLT de $\mathrm{X}$, bien que le contexte discursif relève du spatiotemporel. L'écart construit avec $\mathrm{Y}$ est en effet un mode d'appréhension de $\mathrm{X}$ qui est frappé par le destin, la malchance, le manque de réussite, etc..., étant donné le caractère attendu et valorisé de l'accession à Y qui est déconstruite.

En résumé, l'hypothèse que l'on peut proposer pour SHY est triple :

- Il s'agit d'un marqueur de discernement car il construit une qualification du terme $\mathrm{X}$ à travers sa mise en relation avec le terme $Y$ de par le fait qu'il est conçu comme étant extérieur à Y tout en restant dans son voisinage. Ceci s'explique par une opération de différenciation au niveau de $\mathrm{QLT}_{2}$. Puisque cette opération marque un certain niveau d'identification sousjacente entre les deux termes, il donne la relation $\mathrm{X} / \mathrm{Y}$ comme préconstruite et l'on peut considérer SHY comme une marque de dissociation. Il signale la non-actualisation de leur identification sous-jacente

- Cette différenciation s'interprète comme la cause d'une rupture au niveau de $\mathrm{QNT}_{2}$ dans la mesure où elle construit un hiatus éventuellement quantifiable par un terme $\mathrm{Z}$ entre les termes $\mathrm{X}$ et $\mathrm{Y}$ qui existent de manière séparée et même distancée dans l'espace-temps.

- Etant donné le mode de construction de l'hiatus, celui-ci est relativisé par l'identification sous-jacente jouant entre $\mathrm{X}$ et $\mathrm{Y}$. Il est en fait annulable.

Cette analyse fonde la représentation topologique proposée dans P. Muller (2015a) et dont j'approfondis ici les répercussions théoriques.

\subsection{Représentation topologique de SHY}

On peut donc proposer que SHY donne l'hiatus entre $\mathrm{X}$ et $\mathrm{Y}$ comme annulable et traduirait en fait la différenciation de deux termes au départ pensés comme identifiés l'un à l'autre. L'hiatus quantitatif $\mathrm{QNT}_{2}$ qui les fait exister comme séparés dans l'espacetemps est alors à comprendre comme la conséquence de leur différenciation $\mathrm{QLT}_{2}$. La béance construite à partir de $\mathrm{Y}$ est en fait a priori déconstructible car $\mathrm{X}$ reste identifié à $\mathrm{Y}$ de manière sous-jacente, ce que l'on peut faire figurer dans une représentation 
topologique par un double-ouvert du point de vue de $\mathrm{Y}$ et comme un double-fermé du point de vue de $\mathrm{X}$ :

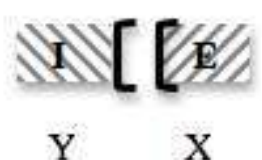

FIg. 1

situation de référence au niveau notionnel, ce qui est congruent avec l'analyse de SHY comme marqueur d' «intentionnalité» sur le plan énonciatif (voir la sous-section suivante).

41 3. Elle autorise, comme on l'a vu, la modélisation d'un recul dynamique, ou même d'une fuite, de la part de X. Ceci permet donc également d'expliquer la possibilité d'un usage verbal de SHY, la distance X/Y pouvant notionnellement varier entre deux instants. La relation entre les deux termes peut d'ailleurs faire l'objet ou non d'une téléonomie ( $\mathrm{P}$. Muller, (2015a)). En (16), les «back-benchers » (X) s'abstiennent consciemment d'annuler la distance qui les sépare de $Y$, une rébellion contre leur propre parti politique. Ils «se (main)tiennent à l'écart » de $Y$ :

(16) [TORY back-benchers $]_{\mathrm{X}}$ last night shied away from [a rebellion over the Budget imposition of value-added tax on domestic fuel $]_{\mathrm{Y}}$. (K5M)

\subsection{SHY comme marque d'intentionnalité} correspond à ce que J.-J. Franckel et D. Lebaud (1990) appellent un marqueur d' « intentionnalité » au sens où la position d'extériorité de $\mathrm{X}$ par rapport à $\mathrm{Y}$ est en fait réexaminée au niveau énonciatif par l'énonciateur qui envisage de manière fictive son entrée dans le domaine de $\mathrm{Y}$.

Si l'on construit le « domaine de validation » associable à la prédication SHY, on obtient le schéma suivant où $\mathrm{X}$ occupe une " position effective » $\mathrm{E}$ extérieure à celle de $\mathrm{Y}$ qui est à l'intérieur I du domaine notionnel. On envisage fictivement l'entrée de X dans I, ce qui passe par la construction intermédiaire d'une position décrochée IE : 


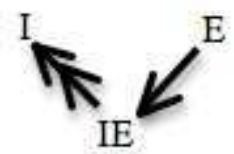

Fig.2

Cette position intermédiaire permet à l'énonciateur d'envisager l'annulation fictive de l'hiatus et d'exploiter énonciativement la continuité sous-jacente entre $\mathrm{X}$ (situé en $\mathrm{E}$ ) et $\mathrm{Y}$ (situé en I).

Ici, l'arrivée en I est considérée comme nécessaire car seul le chemin vers l'intérieur du domaine est pris en compte à l'exclusion de tout autre possible (il s'agit du « fortement unique » évoqué dans A. Culioli (1985: 43)). Autrement dit, l'intentionnalité permet de revenir sur une extériorité de fait et de la réenvisager dans la situation de référence par rapport à l'intérieur du domaine visé rétroactivement. En termes banals, on envisage le fait de ne pas avoir atteint quelque chose mais de toujours se trouver en position de pouvoir l'atteindre, atteinte qui est souvent donnée comme souhaitable. On obtient alors une certaine modalisation de l'énoncé.

L'énoncé (17) illustrera bien le fonctionnement du concept en lien avec SHY OF :

(17) At the quarter-mile post [the Limited $]_{\mathrm{X}}$ is [only $\left.0.3 \mathrm{secs}\right]_{\mathrm{z}}$ shy of [the Integrale's $14.9 \mathrm{secs}]_{\mathrm{Y}}$, but the VW's better aerodynamics and $20.9 \mathrm{mph}$ per 100rpm gearing in fifth allow it to fly to an impressive $139 \mathrm{mph}$ top speed and leave the Lancia flailing in its wake. (A6X)

Il s'agit de dire que le temps mis par la Volkswagen Limited (X) pour faire un quart de mile n'est pas tout à fait celui de la Lancia Integrale $(\mathrm{Y})$ et que l'hiatus qui les sépare est évaluable à trois dixièmes de secondes $(\mathrm{Z})$. $\mathrm{Z}$ permet de quantifier l'écart entre les deux termes dont on a déjà envisagé l'identification possible au préalable car $\mathrm{Y}$ fournit au niveau notionnel la référence spatio-temporelle permettant d'envisager l'extériorité de $\mathrm{X}$ et de la mesurer, ce qui implique que la frontière qui les sépare possède une certaine "épaisseur", conformément à la représentation proposée ci-dessus. De façon intéressante, l'hiatus est encore clairement envisagé comme réductible dans la situation de référence qui n'est pas «close » de ce point de vue, comme le montre le co-texte car la VW va finalement dépasser la Lancia («leave the Lancia flailing in its wake»). Cette possibilité, marquée au niveau notionnel grâce à SHY, est également exploitée au niveau énonciatif. L'énonciateur communique explicitement au co-énonciateur la réévaluation de la position a priori externe de $\mathrm{X}$ par rapport à $\mathrm{Y}$ en donnant la position occupée par $\mathrm{Y}$ comme accessible pour X malgré tout, c'est-à-dire malgré l'hiatus quantitatif. La déconstruction de ce dernier est donnée comme nécessaire dans la situation.

Une telle nécessité aurait pu être exprimée à travers SHORT avec lequel une interprétation de type intentionnalité est aussi disponible mais n'est pas a priori congruente avec le fonctionnement notionnel de cet opérateur, comme on le verra :

(18) [The American fighter $]_{\mathrm{X}}$ crashed just short of [the runway $]_{\mathrm{Y}}$ at Upper Heyford,

killing both men on board. (K1C)

En (18), SHORT OF ne communique pas explicitement que l'avion aurait pu atteindre la piste et ne pas s'écraser mais cette interprétation est néanmoins disponible pour le coénonciateur: l'énonciatif forcerait dans ce cas une réanalyse du notionnel, ce qui peut d'ailleurs correspondre à une stratégie discursive particulière (voir 2.4). On notera par 
ailleurs que des marqueurs comme ONLY en (17) et JUST en (18) favorisent ce type d'interprétation.

$\mathrm{Au}$ niveau notionnel, SHY OF indique en (17) que la VW, dont le temps frise celui de sa concurrente (puisque $\mathrm{Z}$ est quantitativement peu élevé), se trouve encore en position (QLT) de l'emporter dans la situation de référence, c'est-à-dire qu'elle reste en position de combler l'hiatus (QNT). Au contraire, SHORT OF joue d'abord sur QNT et articule deux sous-domaines cloisonnés par une frontière réellement séparatrice, c'est-à-dire strictement fermés, et sans que $\mathrm{X}$ soit appréhendé comme pouvant atteindre $\mathrm{Y}$ à travers la relativisation de cette frontière. Cependant, le concept d'intentionnalité permet de penser une possible réinterprétation en discours de SHORT OF sous un angle qualitatif et de rechercher une identification implicite entre $\mathrm{X}$ et $\mathrm{Y}^{10}$.

\section{L'hypothèse proposée pour SHORT OF}

51 Ayant développé les conséquences théoriques de mes travaux précédents sur SHY (OF), je dispose maintenant d'éléments qui vont me permettre de mieux saisir l'identité de la construction SHORT OF et de SHORT lui-même.

\subsection{Représentation de SHORT OF et analyse en marqueur de "division"}

On peut proposer que SHORT OF et SHY OF marquent un type de hiatus différent, et que le premier marqueur repose sur la mise en place d'une extériorité stricte entre les deux termes $\mathrm{X}$ et $\mathrm{Y}$ par construction d'une frontière séparatrice. L'interruption dans la continuité sous-jacente entre les deux termes concerne cette fois $\mathrm{QNT}_{2}$ et non pas $\mathrm{QLT}_{2}$ :

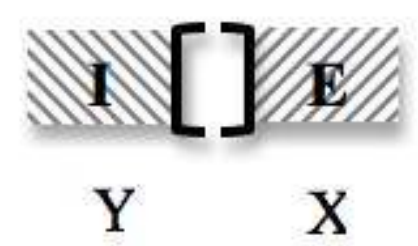

Fig. 3

L'hypothèse proposée est que contrairement à SHY qui pouvait être assimilé à un marqueur de discernement, SHORT OF relève plutôt du marqueur de «division", ce qui « signifie [...] qu'il associe au terme qui [le] suit ([...] noté Y) un domaine sur lequel [il] distingue une ou des « zone(s) » ». (J.-J. Franckel et D. Paillard, 2007 : 7-8).

Il s'agit plus exactement ici de considérer $\mathrm{Y}$ « comme un espace abstrait structurable topologiquement au sens où l'on peut y distinguer différentes zones: intérieur (I), extérieur (E), frontière ( $\mathrm{Fr}$ ) » (D. Paillard , 1998 : 90). On obtient une zone $\mathrm{E}$ associée à $\mathrm{X}$ et séparée de $\mathrm{Y}$, correspondant à I, par une frontière $\mathrm{Fr}$, quantifiable par un terme $\mathrm{Z}$, et qui fait office de coupure "nette » entre les deux termes. Au niveau notionnel, l'enjeu ne réside donc pas d'emblée dans une qualification de X. On a cette fois une différenciation $\mathrm{QNT}_{2}$ (altérité et identification) et une rupture $\mathrm{QLT}_{2}$ (simple altérité) pour X et $\mathrm{Y}$.

La relation marquée par SHORT aurait trois caractéristiques importantes et diffèrerait de celle marquée par SHY en ce que : 
1. L'écart entre $\mathrm{X}$ et $\mathrm{Y}$ n'est pas à considérer à un instant donné comme ayant pu et pouvant varier. Il est stable.

2. Il n'est pas a priori déconstructible et annulable dans la situation de référence à moins que d'autres opérateurs ne viennent ensuite le réduire en le faisant tendre vers zéro ${ }^{11}$.

3. On remarque par ailleurs qu'il ne peut pas facilement évoluer entre deux instants et s'interprète difficilement de manière dynamique. Il n'y a pas d'emploi verbal de SHORT ${ }^{12}$ à moins de rajouter le suffixe -EN qui coderait explicitement cette possibilité. On obtient alors le verbe SHORTEN.

Lorsque c'est le spatio-temporel qui est au centre de la situation de référence, c'est SHORT OF plutôt que SHY OF qui est naturellement utilisé pour en verbaliser la mesure :

(19) [The plane with 20 passengers on board $]_{\mathrm{X}}$ was caught in a strong down draught but managed to touch down safely [ten metres $]_{\mathrm{Z}}$ short of [the runway $]_{Y}$.(K5D).

On perd l'idée qu'autre chose que l'extériorité constatée de X était attendu car l'hiatus quantitatif donné n'est plus déconstructible sous l'effet d'une continuité qualitative sousjacente. L'hiatus est l'enjeu majeur de l'énoncé, alors que SHY OF construisait une distanciation de X par rapport à Y subordonnée à une qualification de X par Y. En (20), il ne s'agit pas, avec SHORT OF, de dire à propos de Stafford Murray qu'on se serait attendu à ce qu'il ait atteint au moins l'âge de 14 ans, étant données ses performances au tennis :

(20) [STAFFORD MURRAY $]_{\mathrm{X}},[19 \text { days }]_{\mathrm{Z}}$ short of [his 14 th birthday $]_{\mathrm{Y}}$, became the youngest winner of a senior county closed championship at the Leominster Leisure Centre, Herefordshire, yesterday when he defeated his coach, Peter Ratcliffe, 9-5, 9-7, 9-2 in the final. (A1N)

Avec SHY OF, même si l'hiatus est quantifiable par un terme Z, ce Z n'est en fait qu'un possible parmi d'autres dans la mesure où l'hiatus est déconstructible, donc variable. La position spatio-temporelle de $\mathrm{X}$ lui-même dans la situation de référence n'est donc aussi qu'un possible parmi d'autres. En (20) qui informe uniquement sur l'âge de Murray, Z n'a pas le même statut et est pensé comme une possibilité unique dans la situation de référence. $\mathrm{La}$ distance $\mathrm{Z}$ qui sépare à un instant $\mathrm{T}$ Murray $(\mathrm{X})$ de son anniversaire $(\mathrm{Y})$ est appréhendée comme stable (i.e. comme si elle n'avait pas varié et ne pouvait pas varier au fil du temps). L'ajout de ONLY semble nuire, si ce n'est à la grammaticalité de l'énoncé, du moins à sa naturalité :

(21) ?[STAFFORD MURRAY] $]_{\mathrm{X}}$, [only 19 days] $]_{\mathrm{Z}}$ short of [his 14th birthday $]_{\mathrm{Y}}$, became the youngest winner of a senior county closed championship at the Leominster Leisure Centre.

Le jugement subjectif marqué par ONLY qui oriente vers l'expression d'un regret entre en conflit avec le contexte, «became the youngest winner... » renvoyant à une valeur unique, positive et valorisée intersubjectivement.

3 De la même façon en (22), il n'était pas spécialement attendu des ailiers qu'ils atteignent la ligne d'essai. Rien d'autre que le maintien de l'hiatus et donc de la position distancée de $\mathrm{X}$ mesurable par Z n'est envisagé dans l'espace-temps. On ne constate a priori aucune modalisation ni prise de position énonciative autre que l'assertion ${ }^{13}$ :

(22) Swansea, whose lead was cut by two penalties by outside-half Mark Thomas, strove to move the ball again in the last 10 minutes with [lock Paul Arnold and replacement Ian Davies $]_{\mathrm{X}}$, at flanker, being checked just short of [the line $]_{\mathrm{Y}}$. (AHK)

d'identification sous-jacente $\mathrm{QLT}_{2}$ entre $\mathrm{X}$ et $\mathrm{Y}$ qui pousserait à relativiser l'hiatus $\mathrm{QNT}_{2}$. Il 
y a une coupure nette de la continuité qualitative qui aurait pu permettre de transcender le zonage spatio-temporel X-Z-Y et autoriser l'assimilation de X à $Y$ en une seule et même entité.

L'emploi de SHORT lui-même comme " combining form » illustre bien ce point. Des noms comme shortbread, shortcake, shortcrust ou même short-manure dénotent des substances caractérisées par leur côté physiquement friable qui s'explique par un manque de cohésion qualitative sous-jacente. Au vu de la rupture $\mathrm{QLT}_{2}$ qui renforce la différenciation $\mathrm{QNT}_{2}$ au niveau intra-notionnel, la notion globale est alors envisageable au niveaux $\mathrm{QLT}_{1}$ et $\mathrm{QNT}_{1}$ comme articulant des éléments discrets et peu cohésifs. La cohésion des notions auxquelles renvoient les noms bread, cake, crust et manure est remise en question par SHORT qui marque donc que l'on ne peut pas vraiment identifier ce qu'ils dénotent à du pain, du gâteau ou du fumier à part entière parce que ces substances jouissent normalement d'une certaine homogénéité interne. La nouvelle notion évoquée est différente car elle ne peut exister dans l'espace-temps de façon durable sans se morceler.

C'est ainsi que l'OED répertorie pour SHORT une première «branche » de sens, résumée par la glose : « having small longitudinal extent » et qu'il explique la collocation short-manure par une impossibilité à s'étendre facilement dans l'espace à cause d'un manque de cohésion interne : «Prob. connected with branch 1 through the notion 'having little length of fibre'" ". Ce qui manque dans l'extralinguistique à cette substance qui n'est " plus vraiment du fumier", c'est un liant. Sur le plan linguistique SHORT, marque justement un morcèlement non compensé par une identification sous-jacente.

\subsection{SHY OF et SHORT OF jouent un rôle différent dans la construction d'une situation de référence}

67 En toute hypothèse, SHY a globalement plutôt à voir avec le discernement qualitatif de X dans la situation de référence tandis que SHORT a plutôt à voir avec le quantitatif, la division de l'espace temps et la distanciation spatio-temporelle de $\mathrm{X}$ par rapport à $\mathrm{Y}$, ce qui se traduit par le fait qu'ils jouent un rôle différent dans la construction que l'on peut faire de la situation décrite. Il s'agira ici de montrer qu'un même contenu référentiel peut être conceptualisé de différentes manières. Je m'inspirerai en cela de R. Langacker (1993 : 447) lorsqu'il oppose "content » et «construal »: «Expressions which evoke essentially the same conceptual content can nonetheless be semantically distinct because they construe that content in alternate ways. »

68 Je fais l'hypothèse que la situation de référence peut être décrite de manière plus ou moins « analytique » ou " synthétique » à partir des instants qui la composent. J'entends par là proposer que son «identité » peut-être appréhendée à travers la prise en compte d'un état ou d'une propriété associée à un seul instant $\mathrm{T}$ ou au contraire nécessiter l'association de plusieurs états ou propriétés à une classe d'instants T. L'énoncé (23), non tiré du BNC mais choisi car il permet de saisir ce point assez facilement, fournira une bonne illustration de la problématique abordée :

(23) Originally the call was short of [the goalline $]_{Y}$, turnover on downs, Indianapolis Colts ball. The booth called a review and referee Jeff Triplette went under the hood. [...] Triplette turned on his mic and said the beautiful words that most Bengals fans didn't expect to hear. Play reversed. Bengals touchdown. (Internet) 

football américain dont l'arbitre a initialement constaté qu'il n'a pas, dans la situation de référence, franchi la ligne de l'embut ( $\mathrm{Y}=$ the goalline) à un instant $\mathrm{T}$. Suivant les règles de ce sport, et puisqu'il s'agit de sa quatrième et dernière tentative pour ce faire, il va donc devoir rendre le ballon à l'équipe adverse, d'où le terme subséquent : «turnover on downs ». SHORT OF est utilisé pour constater de manière objective et irrévocable la position de $\mathrm{X}$ comme extérieure et éloignée de Y.

Ce qui est intéressant c'est que l'hiatus entre $\mathrm{X}$ et $\mathrm{Y}$ marqué par SHORT OF permet de " clore » la situation de référence, de la considérer comme stable (et non plus en cours de stabilisation). Il s'agit pour l'arbitre d'associer un instant $\mathrm{T}$ et une extériorité au sens physique du terme, extériorité dont il ne lui revient pas d'envisager qu'elle puisse varier ou qu'elle ait pu varier précédemment d'un point de vue quantitatif. En fait, l'instant en question est « définitoire » de la situation de référence. L'extériorité de $\mathrm{X}$ par rapport à $\mathrm{Y}$ va induire le passage à une nouvelle situation de référence où la balle va changer de camp. C'est bien ce qui est crucial ici car l'arbitre réévalue ensuite la position effective du joueur (et elle seule) grâce à l'arbitrage vidéo et annule dans les faits l'hiatus marqué par SHORT (d'où la décision " play reversed »). L'arbitre signifie alors que celui-ci a bien atteint la ligne à l'instant $\mathrm{T}$ et la situation de référence réelle devient toute autre que celle constatée au départ: la balle n'est pas perdue, le joueur a marqué et on va passer à une nouvelle situation ou " phase de jeu » puisqu'il va falloir transformer le « touchdown ».

Dans l'analyse ici proposée, SHORT OF ne marque rien d'autre que l'existence d'un hiatus en $\mathrm{T}$ et ne sélectionne qu'une seule position spatio-temporelle pour $\mathrm{X}$ dans l'extériorité pensée par rapport à $Y$. Dans la situation de référence, il permet de fixer la distance qui sépare $\mathrm{X}$ de l'intérieur de $\mathrm{Y}$, et qui peut être quantifiée précisément par un terme $\mathrm{Z}$ comme en (24) décrivant une phase de jeu dans un match de rugby:

(24) It was Dyble who broke the deadlock with a penalty in the first minute and [centre Kelly Wagland $]_{\mathrm{x}}$ came within a whisker of scoring an early try when he stopped [inches] $]_{\mathrm{z}}$ short of [the line $]_{\mathrm{Y}}$. (CF9)

Il ne s'agit pas d'envisager l'hiatus rattaché à l'instant $\mathrm{T}$ comme résultat d'une variation quant à la place de $\mathrm{X}$ par rapport à $\mathrm{Y}$, ni comme suggérant une variation possible à un autre instant $\mathrm{T}$ subséquent. Dans ce type de contexte, la position de $\mathrm{X}$ dans l'espace est ce qui rend la situation de référence, ou la phase de jeu, stable pour les officiels ou le journaliste qui raconte le match après coup, de la manière la plus factuelle possible.

73 Revenons à (23) : la prise en compte d'une seule et unique position possible du point de vue QNT à l'instant $\mathrm{T}$ suffit à clore la situation de référence dans un sport où faire progresser ou non la balle a des conséquences sur la définition des différentes phases du jeu, c'est-à-dire sur l'identité même des situations décrites. Une autre position possible pour X est pourtant envisageable mais pas du point de vue des officiels ${ }^{14}$. Elle pourrait éventuellement correspondre à l'entrée de $\mathrm{X}$ dans le domaine construit à partir de $\mathrm{Y}: \mathrm{X}$ pourrait atteindre $\mathrm{Y}$. C'est pourquoi le commentateur sportif dira très régulièrement «shy of the goalline » au lieu de "short of the goalline ». SHY marque que la possibilité théorique d'une variation caractérisée par l'atteinte du but est implicitement rejetée car elle ne correspond pas aux faits. Le commentateur n'envisage pas uniquement la position effective du joueur mais en fait sa progression contrariée et l'ensemble de ses conséquences possibles pour le match. Son point de vue est différent de celui de l'arbitre 
(25) A fumble would derail [the Bobcats' next possession $]_{\mathrm{X}}$ just [a few yards $]_{\mathrm{Z}}$ shy of [the goal line $]_{\mathrm{Y}}$, but Sanborn would go three and out, their punt only getting to their own 34. (Internet)

SHY permet de « remettre en perspective » la position de $\mathrm{X}$ dans la situation de référence. On notera la présence du verbe «derail $»^{15}$ et celle du modal WOULD qui marque l'inhérence de la relation sujet/prédicat et construit une prédiction (R. Quirk et al., 1985 : 232). SHY marque bien la déconstruction d'une relation préconstruite. L'extériorité de $\mathrm{X}$ est mesurable et rattachée à un instant $\mathrm{T}$ mais sa position spatiale est donnée comme variable au sens où elle n'est qu'un possible parmi d'autres dans la situation décrite, d'abord parce qu'on pourrait imaginer que l'hiatus n'existe pas au même instant et ensuite parce que l'hiatus reste susceptible d'évolution à d'autres instants de la situation, la phase de jeu n'étant pas considérée comme close. On pourrait parler d'effet d'« imperfectivité » avec SHY OF.

La situation décrite est alors implicitement appréhendée du point de vue de son identité à travers une classe d'instants $\mathrm{T}$ dont chacun est associable à d'autres positions spatiales pour X. La situation reste en cours de stabilisation référentielle. Elle reste pour ainsi dire équivoque alors que SHORT OF indiquerait une situation de référence qui ne se laisse définir qu'à partir de l'association d'un seul instant au hiatus spatial constaté, c'est-à-dire de manière univoque. Les autres positions possibles pour $\mathrm{X}$ n'ont aucun statut particulier dans la situation de référence et ne peuvent jouer dans la définition de son identité.

De fait, le terme $\mathrm{Z}$ serait traité différemment : il serait vu avec SHY OF comme une discontinuité constatée dont l'élimination est donnée comme nécessaire dans la situation dont la description reste ouverte. A l'inverse, lorsque la description de la situation est considérée comme arrêtée, l'élimination de Z n'est plus possible et c'est SHORT OF qui est utilisé. La présentation des choses est plus factuelle, moins modalisée. La réalité de la situation ne peut plus être remise en question, notamment lorsqu'on la considère du seul point de vue des statistiques :

(26) A special mention for Ahlah Towns: during the course of ten league matches [she and her parents] $]_{\mathrm{X}}$ have ended up [only eight points $]_{\mathrm{Z}}$ short of [her maximum of 630 points $]_{\mathrm{Y}}$. Statistically, Oxted Green' B' can be denied promotion from division three but it is highly unlikely that they will fall in their last match. (BM4)

Une substitution par SHY OF indiquerait une tendance à "revenir sur le résultat»: différents possibles seraient envisagés à l'intérieur d'une seule et même situation de référence, alors qu'avec SHORT OF on ne donne de statut qu'à un seul possible dans le cadre situationnel décrit. C'est la raison pour laquelle dans ces énoncés en SHY OF, il s'agit souvent, comme le montre le contexte de (27), d'exprimer un regret de l'énonciateur qui prend position par rapport à certaines insuffisances de $\mathrm{X}$ ou son incapacité à atteindre $Y$. On notera le rôle du modal COULD dans cette interprétation, qui marque qu'un autre possible est envisageable :

(27) [Jaguar sold 21,000 cars in the US last year] ${ }_{\mathrm{X}}$, well shy of [the 82,000 sold by Mercedes-Benz and the 72,500 of its German rival BMW] $]_{\mathrm{Y}}$. Saab similarly sells relatively few cars in the US, though its numbers have been rising dramatically. But analysts say better distribution and marketing by either GM or Ford could exploit these valued brand names far more extensively. (A4F) 


\subsection{Modulations contextuelles : Iorsque SHORT OF oscille entre division et discernement}

78 L'hypothèse de l'association de SHY OF au qualitatif et au discernement et de SHORT OF au quantitatif et à la division demande à être nuancée dans la mesure ou une étude des emplois de la séquence SHORT OF va dans ce sens mais montre que les repérages qualitatifs peuvent aussi parfois dominer, au détriment des repérages quantitatifs attendus.

\subsubsection{Quand QNT est privilégié. De l'hiatus à la « portion congrue »}

79 Une première ligne interprétative dégageable pour l'énoncé en SHORT OF en accord avec l'hypothèse générale défendue ci-dessus fait de l'hiatus lui-même l'enjeu principal de la construction, celui-ci étant l'objet d'une évaluation en termes de quantité. Une part importante des énoncés du corpus intègre en effet un terme $\mathrm{Z}$ qui peut être assimilé à une matérialisation en surface de la distance cognitive qui sépare $\mathrm{X}$ et $\mathrm{Y}$. C'est le cas de « one match» en (28) :

(28) $[\text { Bird }]_{\mathrm{X}}$, now 59 , will be [one match $]_{\mathrm{Z}}$ short of [the legendary Frank Chester's record of $48 \mathrm{Tests}]_{\mathrm{Y}}$ ( $(\mathrm{CH} 7)$

On évalue l'étendue de la frontière ou l' « épaisseur » de la coupure qui sépare $\mathrm{X}$ et $\mathrm{Y}$, ce que l'on mettra en valeur sur le schéma en faisant ressortir la béance au niveau de $\mathrm{QNT}_{2}$ :

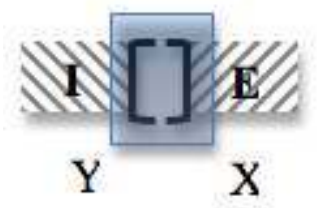

Fig. 4

81 Notons qu'en (28) le terme Z (« one match ») se laisse assimiler à une occurrence extraite de la classe d'occurrences construite à partir de $\mathrm{Y}$ qui constitue le terme de départ du repérage (" 48 tests », autrement dit « 48 test matches ».) Dans d'autres exemples, le terme Y non atteint peut renvoyer à un nombre, à un lieu, à un objectif chiffré, à une somme d'argent, etc... mais $\mathrm{Z}$ n'est pas toujours le produit d'une extraction pensée à partir de Y. En (29) et (30), il renvoie dans l'espace-temps à une « zone intermédiaire » par rapport à $Y$ dont l'étendue est mesurable :

(29) [The plane with 20 passengers on board $]_{X}$ was caught in a strong down draught but managed to touch down safely $[\text { ten metres }]_{Z}$ short of [the runway $]_{Y}(K 5 D)$.

(30) [The 17 trucks $]_{X}$ were halted [25 miles $]_{Z}$ short of [the town of Tuzla $]_{Y}$ while UN aides negotiated for a path to be cleared of mines. (CBE)

On se situe bien du côté de la division et $\mathrm{Z}$ est « la part manquante » pour atteindre $\mathrm{Y}$. $\mathrm{X}$ fait quantitativement office de « portion congrue »:

(31) He would have become the first show jumper to win more than 1 million in prize money: at present $[\text { his total earnings }]_{\mathrm{X}}$ are $[\text { about } 50,000]_{\mathrm{Z}}$ short of $[\text { that }]_{\mathrm{Y}}$. (AJJ) 

jockey en question a dû se contenter de 950,000 livres sterling. En fait, aussi bien Y que Z restent tous deux inaccessibles à $\mathrm{X}$.

A ce stade, on peut faire l'hypothèse, qui mériterait d'être développée ailleurs, que SHORT lui-même, lorsqu'il est prédiqué de $\mathrm{X}$ sans être explicitement mis en relation avec un terme $\mathrm{Y}$, permet précisément de construire $\mathrm{X}$ en portion congrue, c'est-à-dire par élimination de $\mathrm{Y}$ et donc aussi de $\mathrm{Z}$ :

(32) She seems very masculine, with short $[\text { hair }]_{\mathrm{X}}$, jeans and heavy boots and she has a sort of butch look I can't describe. (CH1)

(33) $[\text { The solos }]_{X}$ are short, packed with a powerful energy as if in defiance of his life-threatened status, or to demonstrate a total disregard towards it. (K57)

Le terme $\mathrm{Y}$ implicite serait alors conçu comme une possible extension supplémentaire de $\mathrm{X}$ dans l'espace-temps. Prédiquer SHORT de X, serait une façon de marquer que ce dernier ne saurait être prolongé par Y qui constituerait une continuation de sa propre longueur ou durée. Ainsi, $\mathrm{X}$ serait conçu comme « réduit au minimum » dans la mesure où SHORT marquerait qu'il lui est impossible de s'étendre davantage, le lexème se laissant d'ailleurs définir par « relatively small in extent » (ODE).

Puisque seul le terme $\mathrm{X}$ serait convoqué d'un point de vue référentiel, tout le reste étant relégué au second plan, on analyserait cette fois $\mathrm{X}$ comme l'intérieur du domaine :

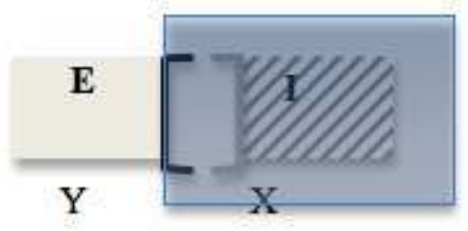

Fig. 5

Le terme Y qui correspondrait à l'extérieur serait éliminé et, par voie de conséquence, le terme $\mathrm{Z}$ également puisque l'extension de la coupure entre $\mathrm{X}$ et $\mathrm{Y}$ ne pourrait plus être quantifiée. L'hiatus n'en serait plus vraiment un et seule serait prise en compte la borne de gauche par rapport à $\mathrm{X}$, qui deviendrait la frontière extérieure marquant la fin de son extension spatio-temporelle :

(34) You'll find the magic kingdom a short [drive $]_{\mathrm{X}}$ off the M6, between Wigan and Preston (M61 junction 8). (CH1)

Ainsi, le chemin qui mène au lieu évoqué en (34) ne saurait se prolonger davantage en Y. Aucune zone intermédiaire $\mathrm{Z}$ n'est conceptualisable et on ne peut saisir la fin de l'extension de $\mathrm{X}$ que par rapport à lui-même : il est, pour ainsi dire, « réduit à sa plus simple expression ».

\subsubsection{Quand QLT prend le pas sur QNT}

On verra ici que l'on s'éloigne de la problématique de la division pour se rapprocher de celle du discernement lorsque :

- -Z marque une appréciation subjective sur l'importance ou la faiblesse de l'étendue de l'hiatus

- -les termes $\mathrm{Y}$ et $\mathrm{Z}$ sont envisagés de manière QLT plutôt que QNT (contrairement au cas précédent) 
- -des marqueurs de modalisation et des adverbes de manière viennent signaler que l'hiatus est aussi un enjeu QLT.

Ainsi, SHORT OF est parfois co-occurrent de termes dont la fonction est de marquer une appréciation de l'énonciateur sur l'étendue de l'hiatus entre $\mathrm{X}$ et $\mathrm{Y}$ et l'on trouve régulièrement les séquences WELL / FAR / JUST / A LITTLE (BIT) / SHORT OF dans le corpus alors que ces marqueurs se combinent difficilement avec SHY :

\begin{tabular}{|l|l|l|}
\hline & Section «newspaper & Ensemble du BNC \\
\hline WELL SHY OF & 0 & 1 \\
\hline WELL SHORT OF & 12 & 60 \\
\hline FAR SHY OF & 0 & 0 \\
\hline FAR SHORT OF & 15 & 128 \\
\hline JUST SHY OF & 1 & 3 \\
\hline JUST SHORT OF & 15 & 82 \\
\hline A LITTLE SHY OF & 0 & 0 \\
\hline A LITTLE SHORT OF & 1 & 10 \\
\hline A BIT SHY OF ${ }^{16}$ & 1 & 2 \\
\hline A BIT SHORT OF & 2 & 13 \\
\hline
\end{tabular}

Dans ces énoncés, la position reculée du point de vue de QNT est pensée comme également significative du point de vue de QLT :

(35) With 609 results declared by 4 am, Labour had made [44 gains] $]_{\mathrm{X}}$, well short of [the advance needed to win power $]_{\mathrm{Y}}$. (AJU)

(36) But in 1979 the Welsh voted four-to-one against a devolved assembly while in Scotland fewer than [33 per cent] $]_{\mathrm{X}}$ supported the idea, far short of [the 40 per cent required by parliamentary decree $]_{\mathrm{Y}}$. (AHX)

Il s'agit, en plus d'apprécier quantitativement la béance qui sépare $\mathrm{X}$ et $\mathrm{Y}$, d'exprimer ce qui est qualitativement une insuffisance de $\mathrm{X}$ dans la situation décrite dans la mesure où Y est valorisé et lui est nécessaire dans la situation. On note ainsi le rôle joué par needed et required en (35) et (36) qui viennent construire l'hiatus constaté objectivement par SHORT OF comme un défaut puisqu'un nombre attendu n'est pas atteint. L'appréciation subjective portée sur X n'est pas construite seulement par SHORT OF mais est un effet du contexte et s'appuie sur d'autres marqueurs. L'hiatus ne cesse pas pour autant d'être pris en considération : il est la cause de la qualification de X par Y (alors qu'avec SHY il en était plutôt la conséquence). On élargit ici la partie de la représentation de SHORT OF qui est mise en vedette car, en plus de l'hiatus, on « braque le projecteur » sur X : 


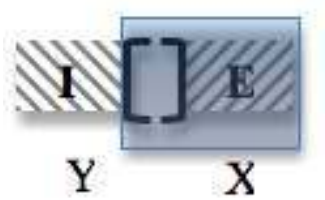

Fig. 6

Le fait pour l'énonciateur de souligner l'importance de l'hiatus permet de basculer vers la problématique du discernement en construisant une position d'éloignement de $\mathrm{X}$ par rapport à $\mathrm{Y}$ qui soit significative par rapport à l'appréhension de son identité qualitative.

En (37), on a le même processus interprétatif mais qui fonctionne de manière inversée. La faiblesse de l'hiatus notée par JUST SHORT OF fait de l'identité de X quelque chose de confus et difficile à décider : on obtient une quasi-assimilation de $\mathrm{X}$ à $\mathrm{Y}$. Ce qui est en jeu du point de vue du discernement est bien de savoir si le placage a eu lieu dans ou hors de l'embut, auquel cas X n'est pas un simple placage (a tackle) mais devient un essai (a try) :

(37) TV commentators often assert confidently that a try has been awarded after [a tackle $]_{\mathrm{X}}$ just short of [the line $]_{\mathrm{Y}}$ because the player's 'momentum' took him over.

(A33)

Ce mécanisme qui lie quantification de l'hiatus et construction d'une qualification de X en fonction d'un terme $Y$ pousse en (38) à relativiser l'échec du cheval Magic Ring qui reste un candidat sérieux ( a most interesting contender ») pour remporter la course car il est seulement A LITTLE BIT SHORT OF Y :

(38) The trainer, who sent out Generous to win two Classics and the King George VI and Queen Elizabeth Diamond Stakes last year, is looking for a replacement and although [Magic Ring] $]_{\mathrm{X}}$ may fall a little bit short of [that high mark] $]_{\mathrm{Y}}$, he is a most interesting contender for the major three-year-old races. (AJJ)

Dans ces exemples, $\mathrm{X}$ est placé dans une position favorable ou détrimentale dans la situation car il est « très loin » ou n'est « pas très loin » d'un objectif qui fait l'objet d'une valorisation par ailleurs. Une traduction par « en mal d'inspiration » le fait bien ressortir en (39) :

(39) [Dynamo Kiev of the newly-independent Ukraine $]_{X}$, sharing some of the problems of the Yugoslav team in that they no longer have a league worth speaking of at home, looked short of [inspiration] $]_{Y}$ against Barcelona in their Group B semifinal. (K4T)

La valorisation contextuelle de $\mathrm{Y}$ en fait un terme dont l'atteinte a priori non effective est analysée comme nécessaire dans la situation décrite. $\mathrm{X}$ devient le point de départ d'une tension sous-jacente mais celle-ci est construite au niveau énonciatif, plutôt que notionnel. L'écart est interprété comme devant être comblé et $\mathrm{Y}$ fait office de point d'arrivée, malgré l'obstacle de l'hiatus : on récupère alors une intentionnalité qui invite à réanalyser SHORT OF comme un marqueur de discernement dans ce type de contexte. On remarque à ce titre la présence d'un MAY de concession en (38) et d'un marqueur de modalisation comme LOOK en (39) (présent aussi en (40) et (45)). En fait, ce type d'interprétation qualitative du marqueur de division est possible car l'énonciateur construit une stratégie discursive par laquelle il invite le co-énonciateur à rechercher et à accepter une qualification de $\mathrm{X}$ à travers l'hiatus avec $\mathrm{Y}$ et ceci malgré le fait que l'on ait SHORT OF et non pas SHY OF. La qualification de $X$ est ainsi "moins directe» puisqu'elle est construite en discours et joue au niveau énonciatif. Elle doit faire l'objet d'un consensus interprétatif entre l'énonciateur et le co-énonciateur, au sens où ce type 
d'effet est anticipable au moment de l'encodage de l'énoncé et que l'énonciateur mise sur le fait qu'il soit compris comme tel au moment du décodage. Il s'agit d'une démarche plus implicative du point de vue de l'interlocution que celle qui consiste à construire directement, au niveau notionnel, une qualification de X à travers SHY $\mathrm{OF}^{17}$. Dans ce cas, l'énonciateur assumerait clairement sa subjectivité et ne compterait pas sur la capacité du co-énonciateur à le suivre dans la construction du jugement sur $\mathrm{X}$.

Cette tendance à l'expression indirecte d'un jugement subjectif (que le co-énonciateur pourrait potentiellement ne pas épouser lui-même, ce qui autorise la polémique) est renforcée lorsque les termes $\mathrm{Y}$ ou $\mathrm{Z}$ sont eux-mêmes envisagés sous un angle plutôt qualitatif. En effet, $Y$ ne renvoie pas toujours à du discontinu, mais peut très bien dénoter des entités plus abstraites qui relèvent du continu comme " genuine championship class " en (40) ou « space » en (41) et pour lesquelles c'est QLT qui domine :

(40) $[\mathrm{NORWICH}]_{\mathrm{X}}$ moved to within a point of the top of the table in a scrappy match against relegation-haunted oldham - but looked a long way short of [genuine championship class $]_{Y^{*}}(\mathrm{HJ} 3)$

(41) Olympic Games: [Olympic village $]_{\mathrm{X}}$ short of [space $]_{\mathrm{Y}}$. (AJJ)

On bascule alors plus nettement encore dans la problématique du discernement car Y désigne une qualité dont $\mathrm{X}$ est privé : Norwich est très loin d'avoir le niveau, le village olympique manque de place, etc... Ce qui est en jeu dans le contexte n'est plus l'hiatus en tant que tel mais son interprétation subjective, bien que sa prise en compte sur le plan QNT reste le point de départ de la qualification. Le tour A LONG WAY SHORT OF en (40) le montre bien (3 occurrences). On trouve aussi dans le corpus « a team SOME WAY SHORT of full strength against Germany " (K5J) (3 occurrences). Notons d'ailleurs que les séquences *A LONG WAY SHY OF et *SOME WAY SHY OF ne sont attestées nul part dans le BNC, ce qui confirme l'idée que l'on a affaire avec SHORT OF, dans ce type de contexte, à un repérage où QLT prend le pas sur QNT alors que SHY OF avait pour raison d'être de coder «plus directement » le repérage QLT.

Cette mise entre parenthèses du quantitatif au détriment du qualitatif est d'autant plus sensible lorsqu'on choisit de ne pas utiliser un terme $\mathrm{Z}$ pour réellement quantifier l'extension de l'hiatus mais que l'on opte plutôt pour un terme dont la fonction première est d'évoquer notionnellement l'idée de finesse. $\mathrm{X}$ est envisagé sur le plan qualitatif comme étant « à un cheveu de... » :

(42) AQUASCUTUM held [ sales $]_{\mathrm{X}}$ only $[\underline{\text { a hairsbreadth }}]_{\mathrm{Z}}$ short of [the corresponding figure $]_{\mathrm{Y}}$ at 23.6 million in the six months to July 31 , but pre-tax profit slid to 616,000 , from 809,000 . (A85)

101 C'est aussi le cas quand $\mathrm{Z}$ renvoie à du quantifiable mais désigne une "quantité négligeable ». On est « à deux doigts » d'avoir la majorité:

(43) $[\text { A few peas }]_{Z}$ short of [a majority $]_{Y}$ ? (AHG)

102 Ce type d'interprétation peut également être corrélé avec l'apparition d'adverbes qui marquent une appréciation plus subjective encore de l'hiatus non explicitement quantifié. On trouve alors par exemple DESPERATELY (7 occurrences dans le corpus) / WOEFULLY SHORT OF (2 occurrences) $)^{18}$ :

(44) But while his second innings batting on Monday looked as though it might save the match, [his bowling] $]_{\mathrm{X}}$ was desperately short of [aggression and conviction] $]_{\mathrm{Y}}$. (K3A)

(45) Walker dropped Mark Robins and brought in Efan Ekoku but [Norwich] $]_{\mathrm{X}}$ looked woefully short of [menace $]_{\mathrm{Y}}$ in attack. (K32) 
Dans le même ordre d'idées, on trouve "SERIOUSLY short of creative ability " (K97), « FRUSTRATINGLY short of magic » (AHG), «SULLENLY short of the natural commodity " (A8N), « TERRIBLY short of money » (K3A). L'adverbe agit alors comme révélateur de la valorisation de $\mathrm{Y}$ évoquée plus haut et des effets de l'extériorité de $\mathrm{X}$, lequel se trouve donc qualitativement défini négativement par rapport à Y.

\subsubsection{Déconstruction QNT de l'hiatus : prépondérance de QLT et centrage sur Y}

Le tour NOTHING SHORT OF, assez courant (24 occurrences dans le corpus) et suivi d'un terme à connotation aussi bien positive que négative, marque une annulation quantitative de l'hiatus. On a alors une mise entre parenthèses encore plus nette de QNT à la faveur de QLT dans le contexte :

(46) [...] [his delirium $]_{X}$ at the end of the first act is nothing short of [hair-raising $]_{Y}$. (A2G)

(47) Compared with the emotionally sterile George Bush [he $]_{\mathrm{X}}$ appears to be nothing short of [a sexual dynamo $]_{Y}$. (CEK)

L'extension de la frontière entre $\mathrm{X}$ et $\mathrm{Y}$ est réduite à zéro et NOTHING SHORT OF permet de centrer sur l'intérieur du domaine construit à partir de $Y$. On a alors une tournure qui signifie REALLY ou COMPLETELY Y. Le tour ??NOTHING SHY OF n'est pas attesté dans le BNC, probablement parce que SHY introduit une coupure qui n'est pas introduite pour être quantifiée mais pour qualifier $\mathrm{X}$.

De façon similaire, SOMETHING SHORT OF (une occurrence), marque la quasi-assimilation qualitative de $\mathrm{X}$ à $\mathrm{Y}$ par minimisation de la distance qui les sépare :

(48) A development of this usage comes in [the form of 'to take a shine to' someone]

$\mathrm{x}$ which indicates something short of [adulation] $]_{\mathrm{Y}}$ (CEK)

Cette logique se retrouve avec LITTLE SHORT OF (13 occurrences) :

(49) They are live Championship contenders - [a transformation $]_{\mathrm{X}}$ which is little short of [remarkable $]_{\mathrm{Y}}$. (CBG)

On trouve ainsi «LITTLE SHORT of miraculous / gasp-inducing / incredible / a disgrace... » traduisibles par « quasi- ou pratiquement $Y$ ». Tous ces exemples marquent une tendance presque effective à l'effacement d'une discontinuité $\mathrm{X}$ par assimilation à $\mathrm{Y}$. Ceci culmine dans un emploi qui repose sur la non-instanciation de $\mathrm{X}$, symbolique de la non-prise en compte de l'extérieur au niveau notionnel. Sans X, la notion même de hiatus, qui impliquait la construction d'une frontière devient caduque et l'on obtient un centrage sur le domaine basé sur Y, à l'exclusion de tout autre possible. SHORT OF Y devient un moyen d'introduire une intériorité notionnelle pour $\mathrm{Y}$ en rejetant et en reléguant dans l'implicite toute extériorité possible caractérisable par un écart par rapport à lui-même. La construction se traduit alors par « à moins de $\mathrm{Y}$ » ou " $\mathrm{Y}$ mis à part " :

(50) Short of a catastrophic breakdown, the chances of that happening were close to zero even before the race started, because Koch is determined to freeze out Conner and make the final an all America Foundation affair. (AKE)

(51) Without those powers, hacking would be almost impossible to prove, short of a confession from the culprit. (A2P)

(52) So, short of sending out an army of class warriors from Teesside, what are we to do with these socially unacceptable socialites? (K4V)

On obtient, dans tous les cas vus dans cette sous-section, une représentation qui met en valeur l'intérieur du domaine construit autour de Y au détriment de l'extérieur. L'hiatus, 
lorsqu'il est pris en compte (énoncés (46) à (49)) n'a qu'un statut secondaire, dans la mesure où l'on pense son élimination.

\subsection{Le " full-deckism », exploitation humoristique de l'interdépendance QNT / QLT avec SHORT OF}

Les points ci-dessus ont permis d'explorer l'idée que la béance quantitative entre $\mathrm{X}$ et $\mathrm{Y}$ est souvent l'objet d'une interprétation qualitative secondaire. La distance qui sépare les deux termes est vue comme révélatrice d'une possible différenciation qualitative. SHORT OF se rapproche alors de SHY OF et s'interprète comme une marque de discernement parce que le couple énonciatif le réanalyse alors comme un marqueur d'intentionnalité. Ce type d'interprétation est exploité dans un type de construction particulier parfois appelé en anglais « full-deckism ».

111 Il s'agit d'une expression censée signifier que quelqu'un est limité intellectuellement, malhabile, ou incompétent. On en trouve de différentes formes et l'on peut par exemple qualifier quelqu'un de «goalie for the dart team » ou bien dire de lui « he plays solitaire for cash $»^{19}$. Elle tire son nom du fait qu'elle exploite l'idée que quelqu'un est mal équipé pour affronter une situation donnée, ce qui est résumable par l'expression «he is not playing with a full-deck». De façon significative, ce type d'énoncé est paraphrasable par « someone isn't all there $»^{20}$. Cette glose construit alors explicitement une qualification pour " someone » à travers l'idée qu'il lui manque certains attributs dans la situation codée par « there ».

On trouve souvent la construction X BE Z SHORT OF Y dans les listes proposées sur internet et le corpus fait apparaitre l'une des plus célèbres de ces formules, « one (or two) sandwiches short of a picnic » :

(53) $[\text { The Government }]_{\mathrm{X}}$, as they say in Glasgow, is [one sandwich $]_{\mathrm{Z}}$ short of [a picnic $]_{\mathrm{Y}}$. So clueless and incompetent, so capable of mismanagement that it has turned it into an art form. (A5D)

La construction de division SHORT OF est à nouveau utilisée dans une problématique de discernement pour $\mathrm{X}$ mais les trois termes impliqués fonctionnent un peu différemment des cas rencontrés jusque-là. Le full-deckism qui suit ce schéma lie en effet l'idée d'incomplétude et de déficience intellectuelle au fait qu'un terme $\mathrm{X}$ est appréhendé qualitativement comme ne disposant pas en quantité suffisante de tel ou tel objet indispensable à la bonne réussite d'un procès implicitement placé au cœur d'une situation de référence évoquée par Y.

114 (53) repose d'abord sur la possibilité pour Y d'évoquer à la fois une classe de situations - le pique-nique - et un procès qui joue un rôle central dans la définition de ces situations : la consommation de sandwichs. La relation notionnelle entre $\mathrm{Y}$ et le terme $\mathrm{Z}$ qui joue le rôle d'argument interne du procès implicite (eat) est une relation préconstruite du point de vue d'une norme interpersonnelle. En fait, dans la réussite du procès sélectionné par la classe de situations, le terme $\mathrm{Z}$ joue un rôle typiquement instrumental. Lors d'un piquenique, on dispose normalement de suffisamment de sandwichs à consommer, ou alors il ne s'agit pas d'un pique-nique.

115 Dans la mesure où $\mathrm{Z}$ spécifie en réalité à la fois la nature et le nombre des artefacts qui font défaut à $\mathrm{X}$ par rapport à une norme situationnelle, il s'agit en réalité d'envisager à travers le «full-deckism » une situation qui est particulière parce qu'elle differe des autres 
situations typiques de la classe évoquée par Y. Ainsi, Z ne permet pas de quantifier l'hiatus qui sépare $\mathrm{X}$ de $\mathrm{Y}$ en tant que tel, mais permet de quantifier l'hiatus qui sépare $\mathrm{X}$ du nombre d'artefacts nécessaire à la réussite du procès placé au cœur de la situation de référence.

X se trouve alors qualifié par cet hiatus : le fait d'être « séparé » d'une situation normale de pique-nique par un sandwich est une manière d'appréhender X comme ne disposant pas de tous les moyens dont il devrait disposer dans la situation. Il s'agit de le disqualifier par rapport à celle-ci et, par extension, par rapport à d'autres situations de référence. Ici, Y peut symboliser toute autre situation dans laquelle se trouve le gouvernement britannique, généralement incompétent aux yeux des Ecossais.

En (54), l'auteur de l'article, intitulé "bun fight ${ }^{21}$ ", utilise une construction de ce type pour signifier que le fait de prétendre prédire l'issue d'un scrutin ( $\mathrm{X}=a$ claim) en comptabilisant la vente de pâtisseries aux couleurs des principaux partis est une idée loufoque, loin d'être pertinente dans une situation d'élection, réputée sérieuse. La situation en question est ici représentée par le mot «teacake » $(\mathrm{Y})$, qui renvoie à un objet dont la confection ${ }^{22}$ suppose que l'on dispose de suffisamment de cassis ( $\mathrm{Z}=$ currants) :

(54) AFTER a decidedly bread and butter campaign, a Darlington bakers is putting its money on hot cross buns. On Thursday, Murrays will offer buns iced in the three main party colours at seven of its shops in the Richmond and Darlington constituencies. By similar random selection, says chairman John Murray, they came' very close' to predicting the 1989 Richmond by-election at 5.30pm on polling day, [a claim $]_{\mathrm{X}}$ that's $[\text { a couple of currants }]_{\mathrm{Z}}$ short of $[\text { a teacake }]_{\mathrm{Y}}$. (K52)

Plus le terme Y sera éloigné de la situation réelle que l'on cherche à symboliser et plus on obtiendra un effet comique. Il est d'ailleurs évident qu'on cherche à créer un effet de style en filant une métaphore alimentaire ( $a$ bread and butter campaign »). On décrit ainsi une démarche qui tranche avec le train-train d'une campagne politique.

En peut également supposer que si l'énonciateur fait appel à SHORT OF plutôt qu'à SHY OF qui, dans mon hypothèse, marque directement le discernement, c'est précisément pour inviter le co-énonciateur à suivre un « parcours interprétatif » qui va du quantitatif au qualitatif :

(55) In its way, it was as funny and arresting as anything from television, but it lacks the scale and presence of a few hundred thousand pounds' worth of television commercial. [It $]_{\mathrm{X}}$ was $[\text { several points }]_{\mathrm{Z}}$ short of $[\text { a pencil }]_{\mathrm{Y}}$. (AJ9)

On vérifiera en effet avec (55) que SHY pourrait théoriquement être substitué à SHORT sans grande différence de sens. Pour autant, les full-deckisms en SHY OF semblent plus rares : aucun exemple n'a été trouvé dans le BNC et la « canonical list of full deckisms » (voir note 20) répertorie visiblement beaucoup moins de constructions en SHY OF qu'en SHORT OF. (56) en constitue pourtant un exemple :

(56) A few links shy of a chain. (http://fuzionbits.com/full_deck.htm)

On peut alors penser que ces full-deckisms pourraient être moins efficaces du point de vue de l'effet comique recherché du fait qu'ils vont en quelque sorte trop directement à l'essentiel, le qualitatif. L'énonciateur gagne en effet à impliquer le co-énonciateur de la plaisanterie en l'invitant à suivre un parcours interprétatif qui oscille entre le quantitatif et le qualitatif, c'est-à-dire entre la division et le discernement. On retrouve l'idée d'une stratégie discursive implicative évoquée dans la sous-section précédente. Le fait que SHORT OF autorise, de manière secondaire une interprétation qualitative de l'hiatus du point de vue de $\mathrm{X}$ permet à l'énonciateur de ne pas assumer directement son jugement 
subjectif, celui-ci reste implicite, et il mise sur la capacité du co-énonciateur à reconstruire ce jugement en discours en réanalysant l'écart $X / Y$ du point de vue de l'intentionnalité. C'est le côté secondaire de ce mode de qualification de $\mathrm{X}$, comparé à celui que construit SHY OF, qui laisse au co-énonciateur une liberté interprétative suffisante qui lui permet de saisir l'humour. On mise sur l'intelligence d'autrui qui doit comprendre qu'il ne s'agit pas vraiment d'un manque de sandwichs ou de cassis mais, implicitement, d'autre chose. Pour être compris, ce type d'humour nécessite une certaine connivence ou complicité du couple énonciatif, ce qui constitue l'enjeu de la stratégie implicative.

\section{Conclusion}

Au terme de ce travail, il apparaît que les éléments théoriques qui avaient été mis en place pour l'analyse de SHY (OF) s'avèrent utiles lorsque l'on cherche à cerner l'identité de SHORT OF. Si le premier lexème privilégie a priori des repérages de type QLT et le second des repérages de type QNT, la réalité des énoncés étudiés montre que SHY peut recevoir une interprétation spatio-temporelle et SHORT OF une interprétation qualitative. J'ai défendu ici l'idée que ce qui était en jeu dans tous les cas au niveau notionnel était la prise en compte d'un hiatus mais que celui-ci était conceptualisé différemment selon le marqueur. Alors que le programme de sens de SHY est de marquer avant tout l'interruption d'une continuité qualitative sous-jacente qui résulte dans l'émergence de l'hiatus, SHORT marque d'abord l'interruption d'une continuité quantitative sous-jacente. Il est d'abord utilisé pour construire l'hiatus, ce qui ne signifie pas que celui-ci et la position que $\mathrm{X}$ occupe par rapport à lui ne deviennent pas souvent également un enjeu qualitatif. J'ai ainsi fait appel au concept d'intentionnalité pour expliquer le fait que le co-énonciateur est en fait souvent invité à rechercher une qualification de $\mathrm{X}$ construite discursivement, auquel cas l'énonciateur l'encourage à s'impliquer lui aussi dans le discernement de l'identité de X. S'il souhaite que la communication réussisse, il mise alors sur sa capacité à réanalyser le marqueur de division qu'est SHORT OF.

\section{BIBLIOGRAPHIE}

Dictionnaires de référence :

Oxford Dictionary of English, $3^{\text {rd }}$ online Edition, Oxford University Press, 2013 (ODE)

Oxford English Dictionary, $2^{\text {nd }}$ online edition, Oxford University Press, 2014 (OED)

Adamczewski, H. et C. Delmas. 1998. Grammaire linguistique de l'anglais. 5 ème édition. Paris : Armand Colin

Culioli, A. 1985. Notes du séminaire de DEA. 1983-1984. Poitiers : D.R.L Paris 7

Culioli, A. 1999a. Pour une linguistique de l'énonciation. Domaine notionnel, T. 3. Paris : Ophrys 
Culioli, A. 1999b. « Des façons de qualifier », in Les opérations de détermination. Quantification/ Qualification. A. Deschamps et J. Guillemin-Flescher, éds. Paris : Ophrys : 3-12

Dufaye, L. 2009. Théorie des opérations énonciatives et modélisation : cheminement d'une réflexion linguistique. Paris : Ophrys

Franckel, J.-J. et D. Lebaud. 1990. Les figures du sujet. A propos des verbes de perception, sentiment, connaissance. Gap : Ophrys

Franckel, J.-J. et D. Paillard. 2007. Grammaire des prépositions. T.1. Paris, Ophrys

Langacker, R. 1993 : « Universals of Construals », in Proceedings of the Nineteenth Annual Meeting of the Berkeley Linguistics Society: General Session and Parasession on Semantic Typology and Semantic Universals. J. Guenter, B. Kaiser et C. Zoll, éds. Berkeley: Berkeley Linguistics Society : 447-463

Thatcher, D. 2014. Saving Our Prepositions, A guide for the perplexed. Friesen Press.

Melis, G. 2004. « Identité, identification et niveaux de qualification : objets et circonstances », in L'Identification, CYCNOS 21. J.-C. Souesme, éd. Nice : CRELA : 77-91

Manning, P. et G. Ray. 1993 : " Shyness, Self-Confidence, and Social Interaction », in Social Psychology Quarterly, Vol. 56, No. 3 : 178-192

Muller, P. 2015a. « Towards a Metalinguistic Representation of the Lexeme SHY » à paraître dans RANAM, 48

Muller, P. 2015b. « Pour un traitement unitaire du lexème anglais SHY », soumis

Paillard, D. 1998 : « Les préverbes russes : division et discernement » in Revue des études slaves, Tome 70, fascicule $1: 85-99$

Quirk, R. et al. 1985. A Comprehensive Grammar of the English Language. Londres, Longman.

\section{NOTES}

1. Le British National Corpus. J'ai choisi de me limiter à la section «newspaper " parce qu'elle offre un nombre d'énoncés raisonnable pour deux lexèmes qui sont extrêmement fréquents en anglais et parce que la construction SHY OF, dans son usage spatio-temporel y est plutôt bien représentée. Cet usage, qui joue un rôle important dans mon analyse, apparaît en effet volontiers dans le commentaire sportif.

2. On sait que la TOPE distingue trois niveaux d'analyse : prédicatif, notionnel et énonciatif.

3. La localisation correspond à un mode de repérage établissant une relation dissymétrique entre deux termes dont l'un sert de repère à l'autre (voir A. Culioli, 1999a : 45).

4. Il s'agit donc d'un mode de repérage lié à QNT dans la TOPE et que je définirai en 1.3, lorsque j'en ferai un usage plus technique et je reviendrai sur le terme de « coupure » en en distinguant deux types, m'appuyant sur les concepts de «rupture» et de "différenciation ». Il s'agira d'opposer les cas où la coupure est envisagée avec ou sans prise en compte d'une continuité sousjacente. Je commence ici à introduire l'idée que SHY marque cette deuxième possibilité au niveau des repérages spatio-temporels.

5. Ce qui va dans le sens d'une relation préconstruite entre $\mathrm{X}$ et $\mathrm{Y}$, voir 1.4 pour d'autres arguments dans ce sens.

6. L'usage qui est fait du mot «coupure » est ainsi plus large que celui qu'en fait A. Culioli qui utilise ce terme, lorsqu'il traite de la temporalité, pour désigner une discontinuité qualitative sans que soit prise en compte une transition entre deux états - auquel cas la coupure s'oppose alors chez lui à la prise en compte d'une frontière (1999a: 165). On verra au point suivant que la 
coupure envisagée ici joue autant sur le plan quantitatif que qualitatif et s'envisage soit sur le mode de la transition soit sur celui de la non-transition, à travers les opérations de différenciation et de rupture.

7. Dans le même esprit, on se souviendra du rôle paradoxal que H. Adamczewski et C. Delmas (1998 : 230) attribuent à of qui fonctionne à la fois comme marque d'interdépendance et comme rupteur.

8. J'utilise le terme «situation de référence» comme synonyme de "situation décrite par l'énoncé » tout au long de cet article.

9. Je défendrai plus loin que SHORT OF s'analyse plutôt au niveau notionnel comme marquant l'interruption d'une continuité sous-jacente de nature quantitative, relevant en cela du marqueur de « division » et non pas de « discernement » (voir 2.1). Si l'on peut récupérer une même nuance de regret avec SHORT OF en (13), celle-ci se construit au niveau énonciatif et n'est pas le fait du marqueur lui-même, ce dont je rends compte au travers du concept d'intentionnalité (voir 1.6).

10. L'énonciateur ne communique donc pas clairement cette possibilité au co-énonciateur mais il peut la laisser entendre : il lui laisse une marge d'interprétation énonciative, c'est pourquoi je parlerai de « stratégie implicative » ci-dessous.

11. Voir en particulier la construction NOTHING SHORT OF Y qui construit un «vidage» quantitatif de l'hiatus. ONLY Z et JUST Z vont aussi dans ce sens. (Voir 2.3.3).

12. A part dans deux contextes assez restreints : 1 . "Faire court-circuit ». "If the contact terminals are shorted, the battery quickly overheats. " et 2. " [with obj.] Stock Exchange. Sell (stocks or other securities or commodities) in advance of acquiring them, with the aim of making a profit when the price falls. "The rule prevents sellers from shorting a stock unless the last trade resulted in a price increase" ". (ODE). Il s'agit en tout cas de marquer l'interruption d'un processus de circulation normale (QNT), du courant électrique ou des échanges financiers, sans que l'on ait de continuité sous-jacente au niveau qualitatif.

13. A moins que l'on récupère une certaine intentionnalité, mais celle-ci n'est pas forcément attendue avec SHORT OF.

14. C'est sans doute pourquoi les arbitres recourent à SHORT et non pas à SHY pour annoncer au public, par micro HF, qu'une équipe fait appel à l'arbitrage vidéo afin de renverser une décision : «Rams challenge the ruling on the field that the ball was short of the first-down marker. " (entendu à la télévision).

15. "Obstruct (a process) by diverting it from its intended course" (ODE).

16. Cette construction ne prend qu'un sens « émotionnel» dans le corpus.

17. C'est cette stratégie qui est exploitée dans le « full-deckism» (voir 2.4)

18. Aucune occurrence de DESPERATELY ou WOEFULLY SHY OF n'a été trouvée dans le BNC.

19. Voir par exemple: http://fuzionbits.com/full_deck.htm et surtout la « canonical list of fulldeckisms » : http://herbison.com/canon/fulldeck.html.

20. http://fuzionbits.com/full_deck.htm

21. " a tea party or other function, typically of a grand or official kind / A heated argument or exchange. " (ODE)

22. Il y a donc là aussi évocation d'un procès typique à travers le mot «teacake ». 


\section{RÉSUMÉS}

Après avoir rappelé l'hypothèse proposée dans P. Muller (2015a) pour le lexème anglais SHY, cet article revient sur l'analyse ébauchée pour SHORT OF à la fin de ce même travail. En faisant appel aux concepts de la TOPE, il s'agit en particulier de défendre l'idée que, contrairement à SHY OF qui marque l'interruption d'une continuité $\mathrm{QLT}_{2}$ entre deux termes X et $\mathrm{Y}$ qui entraîne la construction d'un hiatus dans $\mathrm{QNT}_{2}$, SHORT OF marque a priori l'interruption d'une continuité $\mathrm{QNT}_{2}$ qui est exploitée au niveau de $\mathrm{QLT}_{2}$ dans certains cas seulement. Alors que la première construction pose $\mathrm{Y}$ comme mode d'appréhension qualitatif de $\mathrm{X}$, ce qui en fait un opérateur de " discernement ", la seconde établit un zonage sur le domaine notionnel construit autour de $Y$, ce qui en fait a priori un marqueur de "division». Si les deux constructions diffèrent en ce qui concerne leur analyse au niveau notionnel, la réalité des énoncés où elles apparaissent pousse parfois à les rapprocher dans la mesure où SHORT OF, dans certains contextes, se laisse appréhender au niveau énonciatif comme établissant une certaine " intentionnalité » pour X, ce qui est la norme pour SHY OF. Le marqueur de « division » SHORT OF se laisse alors réanalyser comme marqueur de « discernement ».

After reintroducing the analysis of the English lexeme SHY discussed in P. Muller (2015a), this paper further develops the views on SHORT OF that were only sketched at the end of that previous study. Using the concepts that are available in A. Culioli's theory, it is claimed that SHY OF marks the interruption of a $\mathrm{QLT}_{2}$ continuity between two entities $\mathrm{X}$ and $\mathrm{Y}$, which causes a hiatus in $\mathrm{QNT}_{2}$ to emerge. By contrast, SHORT OF normally interrupts a $\mathrm{QNT}_{2}$ continuity, which is significant regarding QLT $_{2}$ only in some contexts. With the first construction, $\mathrm{Y}$ is construed as a way of grasping the qualitative identity of $\mathrm{X}$ (which makes it an "opérateur de discernement" in D. Paillard's terminology) and the second imposes a zoning of the notional domain built around $Y$ (whereby it can be called an "opérateur de division"). Although the two constructions are analysed differently on the notional level, real examples suggest that they can be brought together on the enunciative level of analysis, since SHORT OF can sometimes be understood as attributing some "intentionality" to X, which is the norm with SHY OF. SHORT OF is then reanalysed on the notional level as marking the same operation ("discernement") as SHY OF.

\section{INDEX}

Mots-clés : shy, short, division, discernement, hiatus, intentionnalité, QNT, QLT

Keywords : zoning, qualitative identity, intentionality

\section{AUTEUR}

\section{PHILIPPE MULLER}

Université Bordeaux 3, Michel de Montaigne

équipe CLIMAS (EA 4196) 